The Astrophysical Journal, 682:602-607, 2008 July 20

(C) 2008. The American Astronomical Society. All rights reserved. Printed in U.S.A.

\title{
EXISTENCE OF THE MAGNETOROTATIONAL INSTABILITY
}

\author{
S. M. Mahajan \\ Institute for Fusion Studies, University of Texas at Austin, Austin, TX 78712; mahajan@mail.utexas.edu \\ AND \\ V. KRISHAN ${ }^{1}$ \\ Graduate School of Frontier Sciences, University of Tokyo, 5-1-5 Kashiwanoha, Kashiwa-shi, Chiba 277-8561, Japan; \\ vinod@ppl.k.u-tokyo.ac.jp \\ Received 2005 August 3; accepted 2008 April 14
}

\begin{abstract}
By posing and solving a global axisymmetric eigenvalue problem on an infinite domain with modes vanishing at zero and infinity for a differentially rotating MHD plasma, the conditions for the occurrence of a purely growing lowfrequency mode known as the magnetorotational instability (MRI) are mapped. It is shown that the MRI criterion drawn from the "local dispersion relation" is at best inadequate and may even be misleading. The physics of the MRI is rather nuanced. It is dictated by the details of the radial profile of the rotation velocity $\Omega(r)$ and not just by the sign and the magnitude of its gradient, $\Omega^{\prime}$. The salient features of the class of profiles for which the MRI-like eigenmodes may occur are given along with the eigenspectrum. For a variety of other profiles, it is shown that an unstable magnetorotational mode is not a valid eigensolution.
\end{abstract}

Subject headings: accretion, accretion disks - MHD

\section{INTRODUCTION}

Accretion disks offer novel and efficient ways of extracting the gravitational energy from compact objects provided the inflow of the matter could be enhanced by the outflow of the angular momentum. Mechanisms causing the augmentation of the angular momentum transport could be provided by the turbulence, hence the search for instabilities. Generally, the shear in the fluid motion is a source of the free energy required to feed an instability, and the accretion disks in the Keplerian motion are well endowed with shear. The shear of the Keplerian flow, however, turns out to be stable against the hydrodynamic instability according to the Rayleigh criterion based on the specific angular momentum $\left[r^{2} \Omega(r)\right]$, where $\Omega=\left(G M / r^{3}\right)^{1 / 2}$. The rotating fluids are stable if the specific angular momentum is an increasing function of the radius (Chandrasekhar 1961) even if the angular speed $\Omega(r)$ is a decreasing function of the radius $\left(\Omega^{\prime}<0\right)$ as it is for the Keplerian rotation profile.

An unsuccessful search for a suitable hydrodynamic instability led, eventually, to invoking the aid of an embedding magnetic field. Could magnetohydrodynamic (MHD) and plasma physical processes provide a possible route to instability - to turbulence?

Although the magnetic stresses produced by the stretching and shearing of the field lines through dynamo-type mechanisms were known to destabilize the system and cause outward transport of the angular momentum (Lynden-Bell 1969; Shakura \& Sunyaev 1973; Blandford 1976; Pringle 1981), it was the pioneering work of Balbus \& Hawley (1991) that brought out the important and essential role of even a very weak magnetic field in exciting an interchange-type linear instability in Keplerian disks. Ever since, there has been a flurry of activity to grasp the essence of the magnetorotational instability (MRI) and its attendant consequences, such as the transport of the angular momentum, by deploying analytical and numerical methods (Papaloizou \& Lin 1995; Balbus \& Hawley 1998). The analytical investigations of the instability to date (Balbus \& Terquem 2001) have been carried out under the so-called local approximation wherein the radial structure in

${ }^{1}$ On leave from the Indian Institute of Astrophysics, Bangalore, India. the cylindrical geometry is either totally ignored or it is treated in a very perfunctory manner. The perturbations are expanded as Fourier modes in the radial coordinate and a kind of radial wavenumber $k_{R}$ is assigned to them. Such a procedure for a nonautonomous system is fraught with danger. It could easily lead us astray, particularly so, if the $\Omega(r)$ profiles have strong radial dependence as the Keplerian $\Omega(r)$ has.

To the best of our knowledge, most of the theoretical work on MRI deals with deriving a criterion for the existence of an instability; the nature and radial dependence of the associated mode has not been addressed. And this is exactly what we propose to do in this paper by investigating the radial eigenvalue problem associated with the MHD equations of a differentially rotating plasma. The eigenmodes we seek are defined over the infinite radial domain; they vanish both at $r=0$ and infinity. Following the existing literature, we also restrict our investigation to the azimuthally symmetric modes ( $m=0$ in the cylindrical geometry). The scope of this analysis is rather broad; it includes, but is not limited to, the Keplerian rotation profile. We study several generic profiles, including some for which the eigenvalue problem is exactly solvable. The primary aim, of course, is to determine if and when a low-frequency purely growing mode such as the MRI exists.

Our results are rather striking. (1) Not all profiles with $\Omega^{\prime}<0$, the consensus "local" criterion for the existence of MRI, lead to an unstable mode; in fact, the mode may not even exist. (2) The Keplerian rotation profile seems to fall into this category. Strictly speaking, therefore, there is no mathematical basis for global MRI-like eigenmodes in the azimuthally symmetric $(m=0)$ standard MHD system. Additional physics (including a model for the rotation profiles in the close vicinity of the compact object, the zero of the domain) will be needed before the question of an unstable mode can be settled. The conventional treatment of the MRI has also been extended to include the nonideal effects such as the Hall effect, the resistivity, and the ambipolar diffusion (Wardle 1999; Balbus \& Terquem 2001; Desch 2004; Kunz \& Balbus 2004). We plan to reinvestigate these effects, in the light of the conclusions of the present study, in the near future. 
Before embarking on a search for growing global eigenmodes, it must be mentioned that unstable eigenmodes are not the only route to turbulence; in a nonnormal (systems with a sheared velocity field), a class of perturbations can have transient amplifications that are large enough that the nonlinear terms can kick in and render the later history different from the predictions of a modal theory. However, such nonmodal effects may play a controlling role when all the eigenmodes are stable; an exponentially growing mode will, in general, dominate the asymptotic behavior.

\section{LINEAR STUDY OF DIFFERENTIALLY ROTATING PLASMAS}

We write the MHD equations in a dimensionless form; all lengths are normalized to an equilibrium length $L$, the magnetic and the velocity fields are respectively normalized to the uniform ambient field $B_{0}$ and the Alfvén speed $V_{\mathrm{A}}=B_{0} /(4 \pi \rho)^{1 / 2}$, where $\rho$ is the uniform mass density, and all frequencies are measured in terms of $V_{\mathrm{A}} / L$. The induction and the force equations then read

$$
\begin{gathered}
\frac{\partial \boldsymbol{B}}{\partial t}=\nabla \times(\boldsymbol{V} \times \boldsymbol{B}), \\
\frac{\partial}{\partial t} \nabla \times \boldsymbol{V}=\nabla \times[\boldsymbol{V} \times(\nabla \times \boldsymbol{V})-\boldsymbol{B} \times(\nabla \times \boldsymbol{B})] .
\end{gathered}
$$

Equation (2) has been obtained by taking the curl of the equation of motion; the pressure term disappears because of the constant density assumption. Equations (1)-(2) allow the equilibrium $\boldsymbol{B}_{0}=$ $\hat{\boldsymbol{e}}_{z}$ and $\boldsymbol{V}_{0}=r \Omega \hat{\boldsymbol{e}}_{\theta}$. We split the fields into their ambient and fluctuating parts,

$$
\begin{gathered}
\boldsymbol{B}=\hat{\boldsymbol{e}}_{z}+\boldsymbol{b}, \\
\boldsymbol{V}=\boldsymbol{V}_{0}+\boldsymbol{v} .
\end{gathered}
$$

Invoking the form of perturbations relevant to the cylindrical geometry,

$$
\boldsymbol{b}=\boldsymbol{b}(r) e^{-i \omega t+i k z+i m \theta},
$$

and using (prime denotes the differentiation with respect to $r$ )

$$
\nabla \times \boldsymbol{V}_{0} \equiv \hat{\boldsymbol{e}}_{z} g(r)=\hat{\boldsymbol{e}}_{z} \frac{1}{r} \frac{d}{d r}\left[r^{2} \Omega(r)\right]=\hat{\boldsymbol{e}}_{z}\left[2 \Omega+r \Omega^{\prime}(r)\right],
$$

the linearized form of equations (1) and (2), in the incompressible limit $(\nabla \cdot v=0)$, turns out to be

$$
\begin{aligned}
\omega_{m} \boldsymbol{b}= & -k \boldsymbol{v}+i r \Omega^{\prime}(r) b_{r} \hat{\boldsymbol{e}}_{\theta}, \\
\omega_{m}(\nabla \times \boldsymbol{v})= & -k(\nabla \times \boldsymbol{b})+i r \Omega^{\prime}(r) \\
& \times(\nabla \times \boldsymbol{v})_{r} \hat{\boldsymbol{e}}_{\theta}-g(r) k \boldsymbol{v}-i g^{\prime} v_{r} \hat{\boldsymbol{e}}_{z},
\end{aligned}
$$

where $\omega_{m}=\omega-m \Omega(r)$.

Equations (4) and (5), coupled with the divergence conditions $(\nabla \cdot v=0$ and $\nabla \cdot \boldsymbol{b}=0)$, can be combined to obtain a single one-dimensional second-order differential equation for the radial component of the velocity or the magnetic field perturbation for any arbitrary value of $m$. We do plan to analyze this general case in the near future. In this paper, however, we only consider azimuthally symmetric perturbations $\left(m=0, \omega_{m}=\omega\right)$. By straightforward algebra, we derive the relatively compact mode equation $\left(\psi=v_{r}\right.$ or $\left.b_{r}\right)$

$L \psi \equiv \frac{d}{d r} \frac{1}{r} \frac{d}{d r} r \psi-k^{2} \psi=-\frac{k^{2}}{F^{2}}\left[4 \Omega^{2}+\frac{2 F}{\omega} r \Omega \Omega^{\prime}(r)\right] \psi$, where $F=\left(\omega^{2}-k^{2}\right) / \omega$ and the definition of $g(r)=2 \Omega(r)+$ $r \Omega^{\prime}(r)$ has been used. Equation (6), in which the mode frequency appears only through $\omega^{2}$, is an exact linear consequence of equations (1) and (2).

\subsection{Special Cases}

Let us now view the mode equation in a familiar setting by dwelling on two of its well-known limiting solutions. Let us begin with the simplest case of rigid rotation, $\Omega=\Omega_{0}$ and $\Omega^{\prime}(r)=0$; the mode equation reduces to

$$
\frac{d}{d r} \frac{1}{r} \frac{d}{d r} r \psi-k^{2} \psi=-\frac{4 k^{2}}{F^{2}} \Omega_{0}^{2} \psi,
$$

allowing a solution

$$
\psi=J_{1}(\mu r),
$$

and the dispersion relation

$$
\left(\mu^{2}+k^{2}\right)=\frac{4 k^{2}}{F^{2}} \Omega_{0}^{2},
$$

describing the Alfvén waves in a rotating plasma. Here, $\mu$ is to be interpreted as an effective radial wavenumber. Notice that in equation (8), the left-hand side represents the square of the total wavenumber comprised of the effective radial wavenumber $\mu$ and the axial wavenumber $k$.

\subsection{Differential Rotation- "Local" Treatment}

Even when the rotation frequency varies with $r$, the conventional treatments set, in equation (6),

$$
\frac{d}{d r} \frac{1}{r} \frac{d}{d r} r \psi=\frac{d^{2} \psi}{d r^{2}}+\frac{1}{r} \frac{d \psi}{d r}-\frac{\psi}{r^{2}}=0,
$$

to arrive at the following "local" dispersion relation,

$$
F^{2}-4 \Omega^{2}=2 r \Omega \Omega^{\prime} \frac{F}{\omega}
$$

or

$\omega^{4}-\omega^{2}\left(2 k^{2}+4 \Omega^{2}+2 r \Omega \Omega^{\prime}\right)+k^{2}\left(k^{2}+2 r \Omega \Omega^{\prime}\right)=0$.

It is straightforward to see that out of the two roots of the quartic, one reduces to the Rayleigh criterion for vanishing magnetic field. The second root represents a purely growing mode, provided

$$
k^{2}+2 r \Omega \Omega^{\prime}<0,
$$

which is possible if $\Omega^{\prime}<0$, i.e., the rotation frequency decreases with distance. This purely growing low-frequency root is called the MRI. The theoretical and experimental pursuit of MRI is a major area of current research.

We contend that this "local" treatment is rather inadequate. In spite of its initial utility, its predictions cannot be relied on. In the nonautonomous differential equation, equation (6), the radial structure of the mode must be dictated by the radial variation of $\Omega(r)$ and cannot be chosen arbitrarily. In fact, different $\Omega(r)$ profiles with the same sign of $\Omega^{\prime}$ could lead to vastly different mode structures with profound consequences for the existence and/or stability of the mode. 
Global analyses of the MRI have been carried out before. Knobloch (1992) derived the governing eigenvalue equation and discussed the stability conditions for different magnetic field geometries and boundary conditions. The limited validity of the local analysis as well as the essential role of the azimuthal magnetic field were emphasized. Dubrulle \& Knobloch (1993) verified the WKB solutions numerically and assessed the relative role of the vertical and the toroidal magnetic fields in the excitation of the MRI. The growth rate was found to be of the order of the Alfvén frequency in the limit of vanishing vertical field. In contrast, Curry et al. (1994) studied the growth rates and the radial eigenfunctions of the MRI for a wide variety of physical conditions pertaining to rotation profiles and magnetic field strengths. They claimed to recover the local behavior with radial eigenfunctions that are sinusoidal in the inner regions and exponentially decreasing in the outer regions. The unstable global mode in a Keplerian disk has been found to reside on the boundaries (Ogilvie \& Pringle 1996). Sano \& Miyama (1999) studied the global MRI including dissipation and the vertical structure by numerically solving the eigenvalue problem. A fully three-dimensional numerical investigation of the linear MRI with boundaries in all directions has been carried out by Rudiger \& Zhang (2001).

More recently, Coppi \& Keyes (2003) investigated the ballooning modes, retaining the global character in the vertical direction but assuming sinusoidal variation in the radial direction. Kersale et al. (2004) find that the wall modes have the properties of the local MRI for boundary conditions that constrain the total pressure at the radial boundaries. Thus, the MRI has remained an active area of research over the years with its simulations (e.g., Hawley 2000), laboratory studies (e.g., Ji et al. 2001) and analytical studies under a host of assumptions. In spite of these many and varied approaches, the global nature of MRI is still far from delineation. The recovery of the local characteristics of the MRI depends crucially on the choice of boundary conditions. The typical boundary conditions, equivalent to walls with infinite potential, will surely define and confine the modes almost independent of the details of the rotation profile. We believe that it is of the utmost importance to learn about the nature of the instability from a boundary-independent (the eigenmodes vanish at the boundaries) analysis; this kind of approach is, perhaps, the key to understanding the effects of different rotational profiles.

\subsection{Differential Rotation-Search for MRI}

Even though the Keplerian accretion disks were the original inspiration for the work on MRI, we deal here with the stability of a generic magnetorotational system represented by equation (6). A general theoretical framework is needed, since the $\Omega(r)$ profiles in laboratory experiments could be far from the Keplerian.

Fortunately, relatively straightforward and well-known analytic methods are enough to extract the relevant physics quite rigorously. It is extremely convenient that the radial dependence of equation (6) (apart from the cylindrical operator) comes from $\Omega^{2}$ and $r \Omega \Omega^{\prime}=(r / 2)\left(\Omega^{2}\right)^{\prime}$. Thus, a model for $\Omega^{2}$ will specify the entire spatial dependence.

\subsection{Exactly Solvable Profile}

We begin our search by investigating nontrivial $\Omega^{2}$ profiles for which the eigenvalue problem, equation (6), is exactly solvable in terms of well-known special functions. For this example, we first manipulate equation (6) to derive an equivalent Schrödingerlike (self-adjoint) equation and then calculate the eigenspectrum; the effective eigenvalue condition will lead us to a dispersion relation involving $\omega^{2}$.
An example of an exactly solvable profile is

$$
\Omega^{2}=\Omega_{0}^{2}\left(1+\frac{\alpha}{r}\right), \quad r\left(\Omega^{2}\right)^{\prime}=-\Omega_{0}^{2} \frac{\alpha}{r} .
$$

For this profile $\Omega^{\prime}<0$ if $\alpha>0$. We have deliberately chosen this form so that the result for rigid rotation can be obtained by simply letting $\alpha \rightarrow 0$. We see below that the problem associated with $\Omega^{2}=\Omega_{0}^{2} / r$ is entirely equivalent, and by a simple transformation, the results obtained for the former can be translated for the latter.

The relevant mode equation

$$
\frac{d}{d r} \frac{1}{r} \frac{d}{d r} r \psi-k^{2}\left(1-\frac{4 \Omega_{0}^{2}}{F^{2}}\right) \psi=-\frac{4 k^{2} \Omega_{0}^{2}}{F^{2}} \frac{\alpha}{r}\left(1-\frac{F}{4 \omega}\right) \psi
$$

is of the form

$$
\frac{d}{d r} \frac{1}{r} \frac{d}{d r} r \psi-p_{0} \psi+\frac{q_{0}}{r} \psi=0,
$$

where

$$
p_{0}=k^{2}\left(1-\frac{4 \Omega_{0}^{2}}{F^{2}}\right), \quad q_{0}=\frac{4 \alpha k^{2} \Omega_{0}^{2}}{F^{2}}\left(1-\frac{F}{4 \omega}\right) .
$$

By the substitutions

$$
\psi=\frac{Q}{r^{1 / 2}}, \quad z=2 \sqrt{p_{0}} r
$$

we can derive the equivalent Schrödinger equation

$$
\frac{d^{2} Q}{d z^{2}}+\left[-\frac{1}{4}+\frac{q_{0}}{2 \sqrt{p_{0} z}}+\frac{(1 / 4)-1}{z^{2}}\right] Q=0
$$

which is the Whittaker equation with $\mu= \pm 1$ and $\lambda=q_{0} / 2 p_{0}^{1 / 2}$. Equation (15) has well-behaved solutions

$$
\psi_{n}=\frac{Q_{n}}{r^{1 / 2}}=r e^{-\sqrt{p_{0}} r} \Phi\left(\frac{3}{2}-\frac{q_{0}}{2 \sqrt{p_{0}}}, 3,2 \sqrt{p_{0}} r\right),
$$

where $\Phi$ is the Kummer function. The solution requires $p_{0}^{1 / 2}>0$ for its existence. The eigenvalue condition

$$
\frac{3}{2}-\frac{q_{0}}{2 \sqrt{p_{0}}}=-n,
$$

then yields the dispersion relation

$$
q_{0}=(2 n+3) \sqrt{p_{0}}
$$

or

$$
\frac{4 \alpha k^{2} \Omega_{0}^{2}}{F^{2}}\left(1-\frac{F}{4 \omega}\right)=|k| \sqrt{1-\frac{4 \Omega_{0}^{2}}{F^{2}}}(2 n+3) .
$$

One immediately notices that the dispersion relation, equation (18), looks very different from the "local" dispersion relation, equation (9); one also sees that the radial quantum number $n$ appears in the spectral relation.

We do not attempt a general analysis of equation (18) for all the modes it allows; we solve it primarily to obtain an approximate 


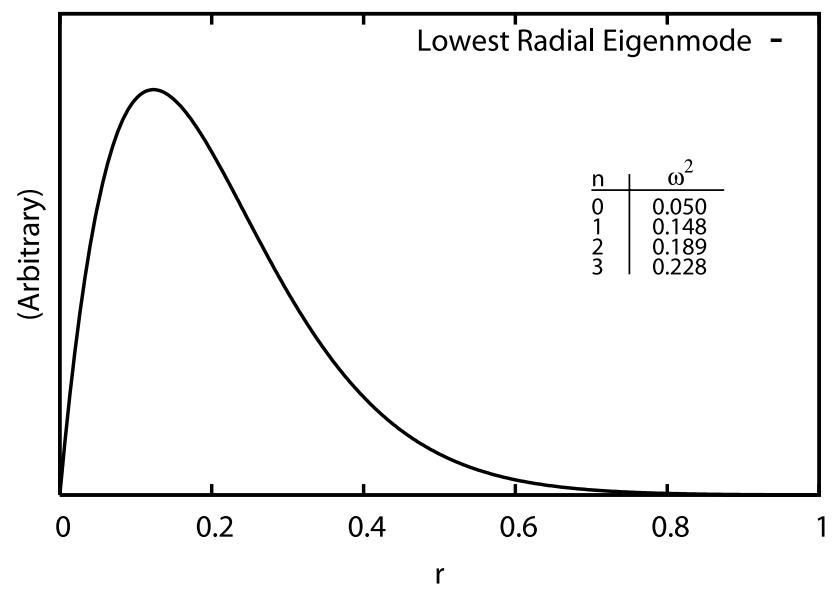

Fig. 1.-Lowest radial eigenmode for profile $\Omega=\Omega_{0}(\alpha / r+\beta r)^{1 / 2}, \alpha=1$, $\beta=14, k=1$, and $\Omega_{0}=4$. Four lowest eigenvalues are listed. The lowest eigenvalue is $\omega^{2}=0.05$ and is stable.

dispersion relation for the MRI-type of mode $\omega^{2}<0,-\omega^{2} \ll k^{2}$. For these conditions,

$$
\begin{gathered}
1-\frac{F}{4 \omega}=1-\frac{\omega^{2}-k^{2}}{4 \omega^{2}}=\frac{3}{4}+\frac{k^{2}}{4 \omega^{2}} \simeq \frac{k^{2}}{4 \omega^{2}}, \\
F^{2}=\frac{\left(\omega^{2}-k^{2}\right)^{2}}{\omega^{2}} \simeq \frac{k^{4}}{\omega^{2}} .
\end{gathered}
$$

With these approximations, equation (18) leads to

$$
\omega^{2}=\left[k^{2}-\frac{\alpha^{2} \Omega_{0}^{4}}{(2 n+3)^{2}}\right] \frac{k^{2}}{4 \Omega_{0}^{2}},
$$

implying that for an instability we require $(\alpha>0)$

$$
\alpha \Omega_{0}^{2}>|k|(2 n+3),
$$

which for small $|k|$, is a much more severe constraint on $\Omega^{\prime}$ (measured by $\alpha$ ) than the local criterion would imply. The existence condition, $p_{0}^{1 / 2}>0$, is trivially satisfied for the unstable root.

As the radial mode number $n$ rises, the instability criterion becomes harder to satisfy; thus, $n=0$ is the easiest to excite. In this case, the instability criterion, although both qualitatively and quantitatively different from the "local" criterion, can indeed be satisfied for $\Omega^{\prime}<0$, and the eigenvalue problem is well posed; well-defined square integrable eigenfunctions are associated with the unstable mode.

The preceding analysis, performed for an infinite system, will clearly not be applicable for a typical laboratory experiment. If the system is bounded $a \leq r \leq b$, then one must take a linear combination of both solutions of the Whittaker equation (15) and determine the dispersion relation by demanding that the total perturbation go to zero at $r=a, b$. Needless to say, the dispersion relation will be considerably more complicated and will require a numerical evaluation.

\subsection{A Simple Numerical Solution}

In this section we present a numerical solution of the eigenvalue problem using the rotation profile $(\alpha, \beta>0)$

$$
\begin{gathered}
\Omega^{2}=\Omega_{0}^{2}\left(\frac{\alpha}{r}+\beta r\right), \\
\left(\Omega^{2}\right)^{\prime}=\Omega_{0}^{2}\left(-\frac{\alpha}{r^{2}}+\beta\right),
\end{gathered}
$$



FIG. 2.- Local criterion $k^{2}+2 r \Omega \Omega^{\prime}<0$ is satisfied for $r<0.265$, but no unstable mode exists.

for which the slope $\left(\Omega^{2}\right)^{\prime}$ can change sign. The purpose is to demonstrate that, although the local criterion

$$
k^{2}+2 r \Omega^{2} \Omega^{\prime}<0
$$

is satisfied in a substantially large region in $r$, there are no unstable modes.

Numerical results were obtained using a finite-difference linear eigenvalue code which can compute multiple eigenvalues simultaneously. Boundary conditions are taken to be Dirichlet at the origin and at a fiducial outer radius. By varying the outer radius so as to ensure that it has no significant bearing on the mode frequencies of the lowest radial eigenmodes, we effectively approximate the infinite domain problem. In particular, the outer boundary condition is well outside the region in which the local instability criterion is satisfied.

In Figure 1 we display the lowest radial eigenmode, along with the eigenvalues for the first few radial eigenmodes. The eigenvalues are all positive; the lowest eigenvalue $\left(\omega^{2}=0.05\right)$ is much larger than the computation error $(\Delta \sim 0.003)$. In Figure 2 we plot the local instability criterion as a function of $r$. Although the local criterion is satisfied for most of the range where the eigenmode amplitude is large, there is no unstable mode. This example clearly demonstrates that the local instability criterion can definitely lead one astray.

\subsection{Effective Potential Approach-General Profiles}

The most important lesson one can learn from the these two example problems is that the eigenvalue problem associated with the magnetorotational system is rather subtle. Relatively crude methods based on the "local" or similar analyses can be seriously misleading. Operationally, the essence of $\S 2.5$ lies in the realization that the existence of a mode of the MRI type, for instance, depends not just on the sign and magnitude of $\Omega^{\prime}$, but on the full radial form of $\Omega$. In addition, a given $\Omega$ may yield a perfectly acceptable solution for $\omega^{2}>0$, while no such solution may exist for $\omega^{2}<0$.

The dethronement of $\Omega^{\prime}$ from its exalted position in the conventional treatment of the MRI is rather difficult to accept; the "local" criterion sounded so patently reasonable. In order to ensure that the exactly solvable cases have not lead us to conclusions that are somehow peculiar and belong to a set of measure zero, let us study the eigenvalue problems associated with classes of $\Omega$ profiles of which the Keplerian will be a particular case. We 
formulate the equivalent Schrödinger-like mode equation for an effective potential due to differential rotation.

Let us assume the rotation profile to have a general power law form

$$
\Omega^{2}=\Omega_{0}^{2}\left(1+\alpha r^{s}\right), \quad r\left(\Omega^{2}\right)^{\prime}=s \alpha \Omega_{0}^{2} r^{s},
$$

where $s$ and $\alpha$ could be positive or negative. Straightforward algebraic manipulations yield the self- adjoint eigenvalue equation

$$
\frac{d^{2} Q}{d r^{2}}+\left[-p_{0}-V(r)\right] Q=0,
$$

where

$$
\begin{gathered}
p_{0}=k^{2}\left(1-4 \Omega_{0}^{2} / F^{2}\right), \\
V(r)=\frac{3}{4 r^{2}}-q_{0} r^{s}, \\
q_{0}=4 k^{2} \Omega_{0}^{2}\left(\frac{\alpha}{F^{2}}+\frac{s \alpha}{4 \omega F}\right) ;
\end{gathered}
$$

the sign of $q_{0}$ is fixed by the quantity in the parentheses.

The general problem of the magnetorotational modes is interesting in its own right, but our current quest is limited to a search for the MRI-type low-frequency instabilities. The eigenvalue problem, equations (25)-(29), has the following interesting features.

1. The eigenvalue $\left(-p_{0}\right)$ is always negative for modes with $\omega^{2}<0$.

2. The potential has two terms. The first one represents the normal cylindrical centrifugal barrier and is always positive. The second term is due to differential rotation, and its sign will crucially determine the nature of $V(r)$.

3. The potential must have a minimum for finite and real $r$ in order to yield a physically acceptable solution. In addition, the $V_{\text {min }}$ must be negative and sufficiently deep so that the effective eigenvalue line $\left(-p_{0}\right.$ with magnitude greater than $\left.k^{2}\right)$ intersects the potential; the intersection defines the turning points that trap the mode. These are still the necessary conditions. The condition of sufficiency will be satisfied if the potential is deep enough to accommodate the zero-point energy associated with the lowest radial eigenmode. That requires an actual solution of the problem. Our attempt, here, is to explore if the potential for a given rotation profile can sustain even a single unstable mode $\left(\omega^{2}<0\right)$.

4. In the range $-2<s, V(r)$ approaches positive infinity as $r \rightarrow 0$. For this genre of potentials, the criteria for MRI can be succinctly summarized as: (a) If $V(r)$ (positive or negative) has no minimum, no mode is possible. (b) If $V(r)$ does have a minimum but $V_{\min }$ is positive, no mode is possible. (c) If $V(r)$ does have a minimum and $V_{\min }$ is negative, then the necessary condition for the existence of a mode is satisfied.

The second term in the potential can be considerably simplified for the low-frequency modes. For $\omega^{2}<0,\left(\omega^{2}-k^{2}\right)<0$, and $\left|\omega^{2}\right| \ll k^{2}$, we have

$$
\begin{gathered}
F^{2}=\frac{\left(\omega^{2}-k^{2}\right)^{2}}{\omega^{2}} \simeq \frac{k^{4}}{\omega^{2}}, \\
F \omega=\omega^{2}-k^{2} \simeq-k^{2},
\end{gathered}
$$

yielding

$$
q_{0} \simeq 4 k^{2} \Omega_{0}^{2}\left(-\frac{\alpha s}{4 k^{2}}\right)=-\alpha s \Omega_{0}^{2}
$$



FIG. 3.-Schematic plots for the potential profiles for positive and negative $s$. The medium-dashed line is for $s=1$ and $\Omega^{\prime}>0$, the small-dashed line is for $s=1$ and $\Omega^{\prime}<0$, the large-dashed line is for $s=-1$ and $\Omega^{\prime}>0$, and the solid line is for $s=-1$ and $\Omega^{\prime}<0$. Since the effective eigenvalue is negative-definite, only the solid profile with negative $s$ and $\Omega^{\prime}<0$ can sustain an unstable MRI-like mode.

We observe that for $s>0, V(r)$ has no minimum for $\alpha<0$ $\left(\Omega^{\prime}<0\right)$ as shown in Figure 3. It does have a minimum for $\alpha>0$ $\left(\Omega^{\prime}>0\right)$, but then $V_{\min }$ is positive (Fig. 3 ). In either case, therefore, no MRI-type mode is possible.

The situation for $-2<s<0$ turns out to be more favorable to the MRI. In this range of $s$, the potential $V(r)$ has a negative minimum (Fig. 4) for $q_{0}>0$, which is clearly possibly for $\alpha s<0$ $\left(\Omega^{\prime}<0\right)$, and the necessary conditions for an MRI-type mode are satisfied. This general result reinforces the conclusion of the exactly solvable case with the rotation profile given by equation (11). We also derived the sufficient condition for the instability for this particular case.

We are thus forced to admit that the mathematical physics behind MRI is a lot richer and subtler than it has been assumed to be so far. Two distinct $\Omega$ profiles with similar $\Omega^{\prime}$ may lead to entirely different consequences; one of these may support an MRIlike unstable mode while the other may not.

We end this section by remarking that results for profiles of type $\Omega^{2}=\Omega_{0}^{2} r^{s}$ are readily obtained from the results for profiles $\Omega^{2}=\Omega_{0}^{2}\left(1+\alpha r^{s}\right)$ by putting $p_{0}=k^{2}$ and $\alpha=1$; there is no qualitative change.

\subsection{MRI under Keplerian Rotation}

We now take the tools of our analysis to the most important MRI-related object - the Keplerian disk for which the rotation profile belongs to the class $\Omega^{2}=A r^{-(2+a)}, a>0$. For the Keplerian disk proper, $a=1$ and $A=G M / L^{3}$, where $L$ is the normalizing distance. The potential in the now familiar eigenmode equation

$$
\frac{d^{2} Q}{d r^{2}}+\left[-k^{2}-V(r)\right] Q=0
$$

has the form

$$
V(r)=\frac{3}{4 r^{2}}-\frac{4 A k^{2}}{r^{2+a}}\left(\frac{1}{F^{2}}-\frac{2+a}{4 F \omega}\right) .
$$

For an MRI-type mode with $\omega^{2}<0$, the signs of the two terms in the parentheses are opposite. If the first term is dominant, then $V(r)$ is positive everywhere and has no minimum and, therefore, cannot support a mode with an effective eigenvalue $\left(-k^{2}\right)$. If the second term is dominant, then the sign of the second term is negative, and the potential goes to negative infinity as $r \rightarrow 0$ (the 


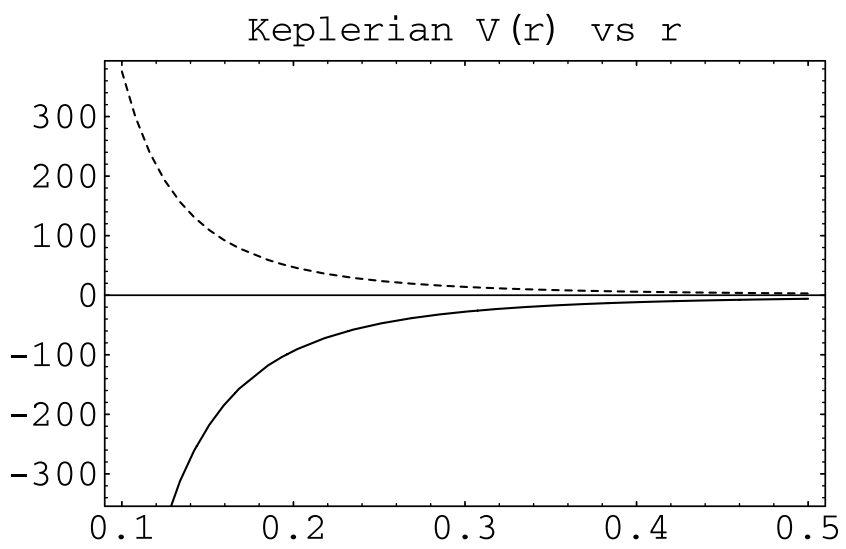

FIG. 4.- Schematic plot of the effective potential for the Keplerian disk. This potential cannot allow any confined states with $\omega^{2}<0$.

Keplerian contribution diverges more rapidly than the centrifugal one for small $r$ ). The total potential $V(r)$ cannot have a minimum, as shown in Figure 3. The purely Keplerian potential then cannot support a bound state with $\omega^{2}<0$. The MRI, considered as an eigenvalue problem on the infinite domain, cannot exist in a Keplerian accretion disk described within the MHD model.

\section{CONCLUSION}

By deriving and analyzing the appropriate eigenvalue problem for a differentially rotating ideal MHD plasma we have shown: (1) For a large class of rotation frequency profiles, including the Keplerian, the conventionally predicted low-frequency, purely growing $\left(\omega^{2}<0\right)$, axisymmetric, MRI-type instability is not possible even for $\Omega^{\prime}(r)<0$; the effective potential stemming from radial dependence of $\Omega(r)$ is found to be inadequate for trapping even the lowest radial eigenmode. (2) For classes of $\Omega(r)$ that do satisfy necessary conditions for the existence of MRI modes, the dispersion relation turns out to be qualitatively different from the one found by a "local" approximation. The local theory, therefore, can often be misleading, in addition to being inadequate. Future analyses as well as simulations of rotating magnetoplasmas must contend with this fact - the MRI they have been looking for may not be there.

We must end this paper with a clarifying remark. This paper does not pass a verdict against an instability in a rotating magnetoplasma, in particular, in a Keplerian disk. We have simply proved that in a minimal model (incompressible MHD, axisymmetric perturbations, uniform embedding field, purely Keplerian profiles extending all the way) one cannot find unstable eigenmodes defined over the infinite domain. We believe that a physicsbased modification of the rotation profile near the compact object is necessary for dependable stability prediction. In addition, the search for the origin of turbulence in accretion disks must be carried to more comprehensible models which include, among other things, compressibility, nonideal and Hall effects, and nonaxisymmetric fluctuations.

The authors gratefully acknowledge many useful discussions with Z. Yoshida, P. Guzdar, and J. Pino; the last two are also thanked for providing the numerical example given in the paper.
Balbus, S. A., \& Hawley, J. F. 1991, ApJ, 376, 214 1998, Rev. Mod. Phys., 70, 1

Balbus, S. A., \& Terquem, C. 2001, ApJ, 552, 235

Blandford, R. D. 1976, MNRAS, 176, 465

Chandrasekhar, S. 1961, Hydrodynamic and Hydromagnetic Stability (New York: Dover)

Coppi, B., \& Keyes, E. A. 2003, ApJ, 595, 1000

Curry, C., Pudritz, R. E., \& Sutherland, G. 1994, ApJ, 434, 206

Desch, S. J. 2004, ApJ, 608, 509

Dubrulle, B., \& Knobloch, E. 1993, A\&A, 274, 667

Hawley, J. F. 2000, ApJ, 528, 462

Ji, H., Goodman, J., \& Kageyama, A. 2001, MNRAS, 325, L1

\section{REFERENCES}

Kersale, E., et al. 2004, ApJ, 602, 892

Knobloch, E. 1992, MNRAS, 255, 25P

Kunz, M. W., \& Balbus, S. A. 2004, MNRAS, 348, 355

Lynden-Bell, D. 1969, Nature, 223, 690

Ogilvie, G. I., \& Pringle, J. E. 1996, MNRAS, 279, 152

Papaloizou, J. C. B., \& Lin, D. N. C. 1995, ARA\&A, 33, 505

Pringle, J. E. 1981, ARA\&A, 19, 137

Rudiger, G., \& Zhang, Y. 2001, A\&A, 378, 302

Sano, T., \& Miyama, S. M. 1999, ApJ, 515, 776

Shakura, N. I., \& Sunyaev, R. A. 1973, A\&A, 24, 337

Wardle, M. 1999, MNRAS, 307, 849 